\title{
MAESTRO DE LA CIRUGÍA CHILENA 2014 DR. JULIO GONZÁLEZ PARDO
}

\author{
Outstanding Chilean surgeon
}

Agradezco al Directorio de la Sociedad de Cirujanos de Chile la honrosa misión de hacer una semblanza del Dr. Julio Fernando González Pardo y rendirle un homenaje al recibir la nominación como Maestro de la Cirugía Chilena 2014.

Julio Fernando nació en Santiago el 21 de octubre de 1939, en el seno de una familia de varias generaciones de maestros primarios, siendo sus dos padres profesores normalistas y su única hermana también profesora e investigadora de Historia. Su padrino y tío político fue el Dr. Kurt Bernhardt, ayudante del Dr. Covarrubias en el Hospital San Vicente de Paul y posteriormente del Dr. Allamand, del cual probablemente captó los primeros atisbos de lo que significaba ser cirujano. Realizó sus estudios secundarios en el Liceo Experimental Manuel de Salas de Santiago, que estaba en esa época bajo la tutela de la Universidad de Chile, adonde llegó a ser Presidente del Centro de Alumnos y egresó con los máximos honores.

Ingresó a la Escuela de Medicina de la Universidad de Chile en el año 1958 realizando gran parte de su formación clínica médico quirúrgica en el Hospital San Juan de Dios y recibió el título de Médico Cirujano en abril de 1965. Durante ese período fue, por un corto tiempo, ayudante de Biología del profesor Gabriel Gasic y participó durante los veranos en trabajos de investigación parasitológica y de Salud Pública en Chiloé, coordinados por la Cátedra de Parasitología del Prof. Amador Neghme. Trabajó como auxiliar técnico en labores médico-quirúrgicas en el Hospital de Victoria en enero de 1963, lugar donde efectuó su primera apendicectomía, gracias a la generosidad del Dr. Rolando Palacios quien iniciaba en esa época su brillante carrera de cirujano y del cual fue posteriormente un buen amigo, apreciándolo como su primer maestro. Fue además ayudante alumno en la Asistencia Pública del Hospital Barros Luco y realizó su práctica como Interno en la Posta 3, en 1964, lugar donde quedó deslumbrado por la vocación y el espíritu docente del Dr. Gonzalo Lopetegui, de quien también fue admirador y amigo toda la vida.

Su primera formación sistemática y formal en clínica médico quirúrgica se forjó al alero de los
Servicios y Cátedras de Medicina y de Cirugía del Hospital San Juan de Dios a cargo de los profesores Rodolfo Armas Cruz e Italo Alessandrini en una época en que en su seno se juntaban grandes figuras de la Medicina Chilena y sus discípulos directos conformaban escuelas de formadores de médicos integrales y preparados tanto para las necesidades sanitarias del país como para la docencia y la investigación clínica. De ese Servicio de Cirugía recuerda con especial emoción y reconocimiento a los profesores Italo Alessandrini y Leonidas Aguirre Mac Kay, a los Jefes de Equipo Drs. Juan Atlagic, Hugo Alesandrini, Jorge León, Daniel Pizarro, Ivonne Pabst y a algunos que fueron especialmente motivantes para él como los Dres. Arturo Jirón, Eduardo Campero, Óscar Contreras, Luis Gautier y Mario Lazo.

Recién egresado ganó un cargo de Médico General de Zona en el Hospital de Río Bueno, X Región, cumpliendo inicialmente 8 meses de orientación y adiestramiento en el Hospital John F. Kennedy de Valdivia, especialmente en obstetricia, cirugía general y urgencias, y atendiendo las postas rurales de Niebla y Corral. Así como lo hicimos muchos médicos en esos tiempos, atendió allí en forma integral todo tipo de problemas de salud, tanto electivos como de urgencia, fue jefe de la Maternidad, concurrió a las Postas de Crucero y Riñinahue en Río Bueno, y cumplió a cabalidad con los Programas de Salud Pública durante esos cuatro años. El contacto con médicos de excelencia, muchos de ellos cirujanos, en el Hospital de Valdivia y en el Hospital de La Unión, y en especial con el Dr. Waldo Pincheira, gran cirujano de Valdivia en esos años, quien con sus enseñanzas, su ejemplo y el apoyo particular que le entregara fueron los factores que más influyeron para que se orientara hacia la difícil, pero hermosa senda de la Cirugía.

En 1969 obtuvo su cupo como becado de retorno para especializarse en Cirugía General en el Servicio de Cirugía del Hospital San Juan de Dios, ahora a cargo del Dr. Luis Figueroa. Por disposición de un programa de regionalización de ese tiempo hizo su primer año en el Hospital Regional de Temuco bajo la tuición de los Drs. Héctor Caviedes y Armin Wes- 
termeyer, centro donde se capacitó, hizo turnos en el Servicio de Urgencia y tuvo la oportunidad de trabajar con un equipo en que se combinaban antiguos y expertos cirujanos con brillantes post-becarios, en su mayoría del mismo Hospital San Juan de Dios, como los Dres. Antonio Baquerizo, Juan Salman, Álvaro Cabello, Luis Burgos y Plácido Flores. Completó su formación básica en la especialidad de vuelta en el Hospital San Juan de Dios, donde profundizó y perfeccionó su preparación clínica, sus conocimientos en patología y fisiopatología, se inició en medicina intensiva y en las técnicas quirúrgicas de avanzada de aquel tiempo gracias a profesores de la talla de los Drs. Santiago Jarpa, Arturo Jirón, Yvonne Pabst, Eduardo Campero, Daniel Pizarro, José Torres, Óscar López y el ejemplo de cirujanos más jóvenes, como William Awad, Robinson Núñez, Roberto Volpato, Jorge Venegas y Hugo Rojas. Allí también compartió y aprendió mucho de sus compañeros de formación, como los Drs. Alarico Escauriaza, Hernán Villagrán, Manuel Barroso, Luis Sierra, Enrique Contreras, Juan Schiller, Harry Schanzer y René Alaff, todos los cuales, años más tarde, brillaron y elevaron el nivel de la Cirugía a lo largo de nuestro país y en el extranjero.

Al término de su especialización en 1972 no se quedó en Santiago, sino que se fue a trabajar al Hospital Roy Glover de Chuquicamata, junto a otros dos ex becarios, bajo la dirección del profesor Dr. Óscar López, lo que les permitió mantener el esquema de trabajo y el mismo espíritu docente asistencial del Hospital San Juan de Dios. Allí hizo turnos de urgencia y de residencia, se hizo cargo de la cirugía infantil en sus aspectos básicos y empezó a focalizarse en la cirugía digestiva alta y baja. Es la época en que hace su primer ingreso a una sociedad científica, la Sociedad Médica de El Loa con un trabajo sobre "Tratamiento quirúrgico de la úlcera duodenal mediante vagotomía troncular y drenaje tipo Jaboulay", y en que realiza su primer bypass intestinal en el tratamiento de una paciente obesa mórbida, que al parecer fue la primera cirugía bariátrica hecha en el país.

Se estableció definitivamente, a comienzos de 1975, en la ciudad de Valparaíso, ingresando al Servicio de Cirugía del Hospital Carlos Van Buren e incorporándose a la Asistencia Pública de Valparaíso. Inicialmente siguió incursionando en amplias áreas de la cirugía general, como la cirugía de cara y cuello, mama y la cirugía venosa, privilegiando eso sí la cirugía abdominal y digestiva, que eran sus preferidas. Colaboró con el Dr. Arturo Villagrán, recién nombrado Jefe de Servicio y de Cátedra, en la modernización del servicio, estrechando lazos con el Servicio de Medicina con el objeto de conformar equipos médico-quirúrgicos especializados, ayudando a crear una Unidad quirúrgica de cuidados especiales, estableciendo las Jornadas Científicas de Primavera anuales, invitando a cirujanos chilenos y extranjeros a operar y dar a conocer los últimos avances en Cirugía, coincidiendo con la vuelta de la docencia de pre y postgrado al antiguo y principal centro hospitalario del puerto, luego del nombramiento del Dr. Arturo Villagrán como profesor titular y de los Dres. Armando Alcázar y Julio Fernando González como sus primeros ayudantes de la Cátedra C de la Escuela de Medicina de la Universidad de Chile, Sede Valparaíso, en 1977.

$\mathrm{Su}$ actividad entonces se concentró en la Cirugía de Urgencia y Digestiva. Se preocupó de revisar la experiencia porteña en esas materias y comenzó a efectuar aportes a nuestra Sociedad de Cirujanos con varias publicaciones acerca de Abdomen Agudo, Traumatismo Abdominal, Pancreatitis Aguda, Hemorragia Digestiva Alta y Complicaciones de la Cirugía Gastroduodenal, ingresando como miembro titular a la Sociedad de Cirujanos en 1983 al relatar su experiencia en la incorporación de la "Desconexión Ácigo Portal con la modificación de Romero Torres en el tratamiento de la Hemorragia Digestiva Alta por Várices Esofágicas e Hipertensión Portal", luego que su trabajo de ingreso fuera revisado y recomendado por el Dr. Exequiel Lira.

Por la formación básica en coloproctología que traía del Hospital San Juan de Dios y la influencia del Dr. Arturo Villagrán, su nuevo jefe en el Hospital Carlos Van Buren, cirujano diestro y muy preparado en la materia, pudo seguir progresando y aprendiendo nuevas intervenciones en el manejo de las megaformaciones, los prolapsos, la rectopatía actínica y el cáncer colorrectal. A partir de 1975 con entusiasmo se incorporó al numeroso grupo de cirujanos digestivos chilenos que siguieron la Escuela Japonesa de estudio y tratamiento del Cáncer Gástrico. Con el Dr. Federico Johow, entre 1980 y 1986 conformó un equipo de cirugía digestiva alta muy productivo, aprendiendo mucho de él en el campo del cáncer esofágico y de toda la cirugía gastroesofágica, lo que les permitió realizar aportes a los congresos y jornadas de nuestra Sociedad y a la literatura quirúrgica nacional, y además colaborar en la formación de grandes cirujanos locales como los Drs. Ignacio Escobar y Sergio Valderrama.

Tal vez su aporte más significativo haya sido el haber sido uno de los pioneros de la cirugía bariátrica en el país al operar su primer bypass yeyunoileal en Chuquicamata en 1974, como tratamiento de una paciente obesa mórbida, con el respaldo y el apoyo del Dr. Óscar López y publicar sus primeras series quirúrgicas en la Revista Médica de Valparaíso en 1982 y en la Revista Médica de Chile en 1986.

Esas publicaciones junto a un caso intervenido 
en Viña del Mar y reportado por el Dr. Jorge de la Fuente en la Revista Chilena de Cirugía en 1975, se adelantaron por muchos años a las provenientes de los centros más importantes de Santiago. En los años 90 inició la cirugía de la gastroplastía y del bypass gástrico abierto. Tardíamente entró a la cirugía bariátrica laparoscópica del banding, de la gastroplastía en manga y bypass gástrico, los que fueron desarrollados mucho mejor por las nuevas generaciones de cirujanos.

Otro aporte trascendental fue su participación en el tratamiento complementario del Cáncer Gástrico, primero con quimioterapia adyuvante con 5-Fluoruracilo y Leucovorina, a comienzos de los años 80 en el Hospital Carlos van Buren con el médico gastroenterólogo Dr. Aldo Lucchini. Con los oncólogos radioterapeutas Drs. Osvaldo Giannini de Valparaíso y Ramón Baeza del IRAM y otros cirujanos el año 1999, en Estados Unidos, presentaron reportes pioneros en Radioquimioterapia Adyuvante en Cáncer Gástrico localmente avanzado y resecado.

Tras el terremoto del año 1985 los Servicios de Cirugía del Hospital Carlos Van Buren y del Hospital Dr. Enrique Deformes se fusionaron al Servicio de Cirugía del Hospital Valparaíso, ahora Hospital Dr. Eduardo Pereira, ubicado en el Cerro Delicias de la ciudad-puerto, integrándose los tres servicios en ese hospital bajo la dirección del profesor Dr. Jaime Venezian. El Dr. Julio Fernando González se integró al equipo de cirugía digestiva de ese nuevo gran Servicio llegando a ser uno de sus pilares, especialmente en el área docente. En ese hospital estuvo a cargo de la creación del Comité Científico, organizó las Jornadas Científicas y además encabezó la primera Unidad de Manejo del Dolor y Cuidados Paliativos.

El año 1991 formó parte del pequeño grupo de cirujanos porteños que inició la cirugía laparoscópica de la vesícula biliar en la región de Valparaíso, a menos de un año de estas primeras operaciones realizadas en Chile, teniendo la suerte de haber participado en la primera colecistectomía laparoscópica regional que se efectuó en el ahora desaparecido Hospital Alemán del cerro Alegre de Valparaíso, y que contó con la gentil colaboración del Dr. Jorge Larach.

Sin embargo, su actividad más destacada y constante desde que llegó a Valparaíso fue la docente. Habiendo tenido ya la experiencia de haber sido secretario de la Unidad Docente de Cirugía en el Hospital San Juan de Dios en 1971, se incorporó en 1977 a la enseñanza de alumnos, internos y luego becados de Cirugía de la Escuela de Medicina de Valparaíso en el Servicio de Cirugía del Hospital Carlos Van Buren y muy especialmente en su Unidad de Emergencia, donde fue Jefe de Turno durante 15 años. En 1978 fue nombrado Profesor
Ayudante, en 1988 ascendió al grado de Profesor Adjunto y en 1997 alcanzó la jerarquía académica de Profesor Titular luego de presentar una Tesis sobre "Tratamiento Quirúrgico del Cáncer de Esófago", con un informe del profesor Dr. Exequiel Lira, autoridad nacional en la materia. Fue consejero de la Facultad de Medicina, miembro permanente de la Comisión Curricular en 1998 y de la Comisión de Acreditación de la Escuela en el 2000, cuya labor permitió que la Escuela de Medicina de Valparaíso fuera la primera en ser acreditada en Chile. En el año 2001 formó parte de la Comisión de Acreditación de Centros y Programas de Formación en Cirugía de la ASOFAMECH. Ese mismo año fue elegido como Profesor de la Cátedra A de Cirugía y Director del Departamento de Cirugía en reemplazo del Dr. Jaime Venezian quien se acogió a retiro.

Numerosos fueron sus alumnos e internos de Cirugía General en sala y en consultorio que se beneficiaron de sus clases y pasos de Cirugía Abdominal y Digestiva.

Muchos fueron sus internos y becados a los que hizo docencia directa en boxes de urgencia y pabellones quirúrgicos, transmitiéndoles sus experiencias de médico general, de cirujano general y de especialista cirujano digestivo, su visión integral de la Medicina y de la Cirugía y su bagaje de valores éticos. Hace 4 años y después de 32 años de meritoria labor se retiró de la docencia universitaria para dejar el paso a las nuevas generaciones. En el año 2012, es nombrado Profesor Emérito de la Facultad de Medicina de la Universidad de Valparaíso.

Durante todos estos años de ardua labor docente asistencial desarrolló una importante actividad científica y de extensión en varias sociedades médicas, siendo la más importante en nuestra Sociedad de Cirujanos de Chile de la que fue director en tres períodos; la Filial V Región de esa misma Sociedad de la cual fue uno de sus fundadores, junto al Dr. José Klinger, en 1986, siendo su presidente en tres ocasiones, y el American College of Surgeons al que ingresó en 1993. Fue un entusiasta y perseverante asistente y participante a la mayoría de los Congresos de la Sociedad de Cirujanos de Chile y del American College of Surgeons a partir de 1965; asistió a numerosos cursos en Chile, Argentina y España siendo incorporado como Miembro Honorario extranjero a la Asociación Médica Argentina y como Miembro Académico Honorífico a la Real Academia de Medicina de Granada en España; tiene unas 50 publicaciones en revistas chilenas, latinoamericanas, una en Estados Unidos y es coautor de un libro de nuestra Sociedad.

Además, el Dr. González, desarrolló algunas actividades gremiales importantes, como haber sido Secretario de la Asociación de Médicos Becarios en- 
tre 1971 y 1972, representante de esa Asociación y del Colegio Médico de Chile al II Congreso Latinoamericano de Residentes en Buenos Aires en 1972 y organizador del III Congreso de Residentes Latinoamericanos en Chuquicamata el año 1973. Años más tarde, en 1982, fue socio fundador de la Asociación Gremial de Cirujanos de la V Región de Valparaíso y secretario durante dos períodos. Finalmente, desde 2012, es miembro del tribunal de Ética del Consejo Regional Valparaíso del Colegio Médico de Chile y acaba de ser nombrado Presidente del Comité de Ética de esa institución.

En cuanto a su vida personal Julio Fernando está casado con Alicia Mella Gajardo, enfermera universitaria, con quien ha constituído una feliz familia de cuatro hijos, Claudia, Ingeniero Comercial y Rodrigo, graduado en Negocios, radicados en Estados Unidos; Pablo, Oncólogo Radioterapeuta y José Miguel, Ingeniero Civil Bioquímico que ejercen en Chile, los cuales le han dado, hasta el momento, ocho hermosos nietos.

Creo que el Dr. Julio González Pardo ha sido un médico integral y un cirujano general clásico, fundamentalmente clínico, formado enteramente en Chile, especialmente comprometido con su país y sus organizaciones sanitarias, que a través del diagnóstico adecuado y del tratamiento razonable y oportuno, ha resuelto aunque a veces sólo ha aliviado, o simplemente ha acompañado brindando su apoyo a los pacientes y a sus familiares que depositaron su con- fianza en él, en su experiencia, experticia, capacidad, bonhomía y espíritu solidario.

Pienso que, además, ha cumplido con creces el mandato hipocrático del médico como docente en todo momento, frente a sus pacientes, sus familiares y su entorno, con el resto de sus colegas, pero especialmente con sus alumnos y médicos en formación, por lo que con justicia se hace merecedor del título de Maestro de la Cirugía Chilena.

Llama la atención que habiendo sido de Santiago y que habiéndose formado fundamentalmente en la capital haya desarrollado la mayor parte de su vida profesional como cirujano y profesor en provincia, lo que constituye una loable excepción que nos muestra un camino diferente y resulta un gran estímulo para los que vivimos en regiones.

Más que las distinciones recibidas al final de su carrera es la satisfacción del camino recorrido, el afecto y el reconocimiento de sus pacientes y discípulos, y el amor de sus familiares y amigos más queridos lo que verdaderamente lo honran, lo enaltecen y lo hacen feliz.

Dr. Juan Bombin $\boldsymbol{F}$. Profesor Titular de Cirugía Universidad de Valparaíso

En Valparaíso, noviembre de 2014

\section{Discurso de agradecimiento de la distinción como Maestro de la Cirugía Chilena}

Dr. Mario Uribe, Presidente de nuestra Sociedad de Cirujanos, respetados miembros del actual Directorio, estimados compañeros y amigos de esta querida institución, señoras y señores: quisiera iniciar estas palabras recordando que en un día como hoy, hace exactamente 50 años, nuestra Sociedad de Cirujanos se reunió para otorgar, con toda justicia, el título de Maestro de la Cirugía Chilena, año 1964, al profesor Dr. Italo Alessandrini. Para mi resulta especialmente emocionante y significativo evocar que en aquel momento y luego de cinco años de haber sido uno de sus alumnos, daba término a mi Internado de Cirugía en el Hospital San Juan de Dios de Santiago y, junto a mis compañeros, me despedía de él con mucho afecto. No sabía en ese momento que seríamos sus penúltimos Internos ya que pocos meses después, a mediados de 1965, iba a fallecer.
Pero muchísimo menos habría podido imaginar, que después de 50 años, uno de sus imberbes internos siguiendo su ejemplo y habiendo recorrido también ese largo, hermoso y a veces pedregoso camino de la Cirugía, pudiera llegar a tener el honor de ser considerado merecedor de similar distinción.

Conmovido, sorprendido y ciertamente muy agradecido por la bondadosa distinción acordada por el Directorio de la Sociedad que preside el Dr. Mario Uribe traté de entenderla y como primer paso me apresuré a dar una mirada a la historia de nuestra Sociedad y al aporte hecho por cada uno de sus maestros al desarrollo de la Cirugía Chilena. Con sólo recordar a varios de ellos que fueron mis maestros directos y constatar quienes habían sido a su vez sus antecesores, varios de ellos figuras señeras de la Cirugía Chilena, he quedado consternado. A través del testimonio de sus discípulos, del registro de sus obras y de la contemplación de fotografías de sus figuras distinguidas, en las que se puede apreciar su 
apostura, prestancia y caballerosidad, ciertamente he sentido que mis méritos resultan escasos ante los de ellos.

Necesariamente tendría que pensar que los tiempos han cambiado y que con generosidad esta Directiva ha querido más bien representar en mi persona el reconocimiento al papel jugado por innumerables colegas y amigos que en estos últimos cincuenta años entregaron su vida al servicio del Sistema Nacional de Salud y a las Universidades Regionales a lo largo de todo Chile y que a pesar de haber estado condicionados y limitados por los avatares de una época muy compleja, de cambios vertiginosos, y de constante reemplazo de paradigmas técnicos, científicos, organizacionales y culturales, tanto en el país como en nuestros sistemas de salud, siempre han estado dispuestos a seguir entregando sus conocimientos y aportando su experiencia a los más jóvenes.

A los cirujanos del último tercio del siglo veinte y primeros años del siglo veintiuno nos correspondió vivir drásticos cambios políticos nacionales e internacionales, revoluciones culturales con grandes consecuencias éticas y conductuales y enfrentar, participando en alguna forma, en el desarrollo exponencial de la ciencia y la tecnología. De una Medicina fundamentalmente clínica y personalizada, todavía con influencia europea, fundamentalmente francesa y alemana, que alcanzamos a aprender y a valorar de nuestros maestros, entramos de lleno a una Medicina cada vez más tecnológica y despersonalizada, especialmente de influencia norteamericana, que nos hizo sin duda ser más eficaces, rigurosos y exactos, privilegiando la especialización y la subespecialización, pero que al mismo tiempo nos hizo menos integrales, más individualistas y más competitivos.

En lo personal he vibrado con entusiasmo por los avances del conocimiento y de la técnica quirúrgica y he tratado de estar siempre al día, pero al mismo tiempo me he esforzado por no perder el antiguo y maravilloso arte de la semiología clínica y la buena anamnesis. Con prudencia y moderación he ido incorporando en mi quehacer médico los nuevos aportes imagenológicos, endoscópicos y de laboratorio en el diagnóstico tratando de no exponer a los enfermos y a los servicios de salud a un desmedido incremento de los costos y los tiempos de espera que producen segregación e inequidad. Igualmente he tratado de mantener y transmitir a mis alumnos el espíritu solidario de los años 60 y 70, que se expresaba muy bien en el Servicio Nacional de Salud de aquel entonces. He luchado porque los cirujanos sigan siendo capaces de entender y considerar la problemática psicosocial de sus pacientes cumpliendo con el imperativo hipocrático de buscar su bienestar físico y espiritual. Y que sean, además, respetuosos y leales con los colegas y todos los miembros de los equipos de salud.

Confieso que en mis primeros 15 años de cirujano me sentía profundamente orgulloso, comprometido e identificado con la escuela quirúrgica del San Juan de Dios de los años estelares de los 60 y 70, que dirigía el profesor Italo Alessandrini desde su llegada al nuevo hospital en 1954. Sentía que pertenecía a la estirpe quirúrgica que venía de los profesores Lucas Sierra y Vargas Salcedo, sus maestros, y que continuaba con la pléyade de grandes cirujanos formados a su alero en ese gran centro docente asistencial, todos los cuales fueron mis maestros directos.

Sin embargo, la experiencia de haber transitado por diferentes regiones y por hospitales de distinta complejidad y de haber conocido a médicos de distinto origen y formación me sirvió para tomar conciencia que no sólo en la capital había buena medicina, que no sólo de mi Universidad salían buenos médicos y que el San Juan de Dios, mi gran centro formador, no era necesariamente siempre el mejor. Rápidamente conocí y valoré tremendamente a los egresados de la Universidad de Concepción y de la Universidad Católica y me identifiqué con las nuevas Escuelas de Medicina de Valparaíso, Valdivia y Temuco con los cuales compartí enseñanzas y experiencias y en las cuales conocí y gané magníficos amigos.

Mi incorporación a la Cirugía porteña, adonde terminé de desarrollarme y donde finalmente pude hacer mis mejores aportes docente-asistenciales, fue especialmente enriquecedora al tener la oportunidad de conocer mejor a sus grandes figuras de antaño, como los Drs. Guillermo Münich, Adolfo Reccius, Romeo Cádiz y José Bengoa; apreciar la tradición de calidad de los hospitales Gustavo Fricke, Carlos Van Buren, Enrique Deformes, Naval Almirante Nef, Alemán y de nuestro último hospital Dr. Eduardo Pereira, donde se unificaron los tres servicios de cirugía de Valparaíso en 1986; de conocer y valorar la calidad y el aporte de las más notables figuras de la generación que me antecedió como los Drs. Óscar Zunzunegui y Arturo Villagrán del Carlos Van Buren, Carlos Pattillo y Rodrigo Manubens del Enrique Deformes, Svänte Tornvall, Pedro Uribe y Jaime Venezian del Hospital Valparaíso, Elías Bitrán y Jorge Kaplán del Hosp. Naval y Jorge y Hernán de la Fuente, José Klinger y Miguel Orriols del Gustavo Fricke, todos ellos maestros respetables y reconocidos y cuya capacidad como directivos y jefes de servicio permitió avances quirúrgicos notables y el desarrollo de grandes iniciativas regionales como la creación de la Escuela de Medicina de Valparaíso. Rindo homenaje y agradezco a todos ellos por las oportunidades y enseñanza directa o indirecta que me dieron en el servicio, en la docencia y en las 
actividades de las sociedades científicas y gremiales regionales.

Considero que es todo un hito que después de 22 años un cirujano de provincia vuelva a ser elegido Maestro de la Cirugía Chilena, tomando en cuenta, además, que han sido poquísimos los que la recibieron: César Garavagno de Talca, Ignacio González, que era sólo en parte de Concepción y mis antecesores de Valparaíso Guillermo Münich, Svante Törnvall, Pedro Uribe y Jorge Kaplán. Conociendo la sacrificada y trascendente labor de los cirujanos en los centros regionales y sabiendo el afecto y el prestigio que algunos han tenido en sus comunidades y entre sus discípulos, cuesta entender que en ese grupo no hayan estado presentes algunos insignes cirujanos de Iquique, Antofagasta, La Serena-Coquimbo, Rancagua, Talcahuano, Temuco, Valdivia o Puerto Montt.

Quisiera destacar, también, que después del Dr. Roberto Burmeister, es la segunda vez que un cirujano que ha hecho la carrera larga, comenzando como Médico General de Zona, completado su formación formal como Residente Becario de Retorno o Secundario y haya sido cirujano de Urgencia y Jefe de Turno gran parte de su vida recibe esta distinción. Y, sin embargo, hemos sido muchos, y con los mismos méritos, los formados en ese modelo, que privilegiaba la formación primero como médico integral básico, luego como especialista general y sólo finalmente como subespecialista y lo han hecho bastante bien.

Es verdad que este es un modelo actualmente cuestionado porque presupone que los futuros cirujanos tendrían que conocer la disciplina e introducirse en su práctica desde el pregrado y, no como se plantea ahora, como actividad estrictamente de postgrado. Es cierto también que ese esquema va en contra de la tendencia moderna a una especialización precoz la que por lo demás nos tiene bastante en jaque por la demanda irracional de especialistas por la población, por las expectativas un tanto egoístas de muchos médicos jóvenes, por la incapacidad de las escuelas formadoras en aumentar la oferta y la impotencia de los servicios de salud de dar cobertura y calidad en la salud pública. ¿No es verdad que en todas partes del mundo, incluyendo Estados Unidos, se ha estado revalorizando el papel del cirujano general en la comunidad, en la atención primaria y secundaria, en la docencia de pregrado y como indispensable primera etapa en la formación de postgrado? y ¿no es cierto que la ultraespecialización, consecuencia del deseable y necesario desarrollo de la técnica, parece llevarnos a niveles de virtuosismo tales que podrían amenazar en el futuro a convertir a los cirujanos en tecnólogos altamente calificados olvidándose de la esencia de la Medicina?

Ahora sé que pertenezco a la gran escuela chilena de cirujanos, que está indisolublemente unida a la historia de esta Sociedad y que es secundada por el Capítulo Chileno del American College of Surgeons y apoyada por los Departamentos de Cirugía de las Universidades Chilenas. Reconozco en la Sociedad de Cirujanos de Chile a mi principal fuente de educación y formación continua en la segunda parte de mi vida, al igual que de la inmensa mayoría de los cirujanos de este país. Ella nos ha dado la oportunidad de conocer y relacionarnos, con personalidad e independencia, con lo mejor y más avanzado de la Medicina y de la Cirugía nacional y extranjera. De ello me siento muy agradecido. En su seno hace tiempo que me siento entre hermanos y amigos. Ella es mi familia quirúrgica.

Quisiera terminar estas palabras expresando que en mi vida la mayor recompensa ha sido tener una maravillosa familia y que mi principal apoyo en los buenos y en los malos momentos ha provenido de la fortaleza, de la comprensión y del amor que me ha dado Alicia, mi querida esposa. A ella, a mis hijos, a mis nietos y a mis padres que ya no están, dedico esta honrosa distinción.

Dr. Julio Fernando González Pardo 